\title{
3D Printing for Energy-Saving: Evidence from Hydraulic Manifolds Design
}

\author{
Jun-hui Zhang ${ }^{1, * \odot}$, Gan Liu ${ }^{1}$, Ruqi Ding ${ }^{2}$, Kun Zhang ${ }^{1}$, Min Pan ${ }^{3}$ and Shihao Liu ${ }^{1}$ \\ 1 State Key Laboratory of Fluid Power and Mechatronic Systems, Zhejiang University, \\ Hangzhou 310027, China \\ 2 Key Laboratory of Conveyance and Equipment, Ministry of Education, East China Jiaotong University, \\ Nanchang 330013, China \\ 3 Department of Mechanical Engineering, University of Bath, Bath BA2 7AY, UK \\ * Correspondence: benzjh@zju.edu.cn; Tel.: +86-571-87952505; Fax: +86-571-87952507
}

Received: 24 May 2019; Accepted: 23 June 2019; Published: 26 June 2019

\begin{abstract}
With the compact circuit layout and small size, hydraulic manifolds sometimes cause high pressure loss. The purpose of this paper is to investigate the pressure loss under different circumstances with various geometry features and present solutions to reduce pressure loss. The pressure loss performance is evaluated by both experimental and numerical methods. Verified by the experiments, the numerical simulations are qualified to depict the correct trend of the pressure drop. After the basic analysis of traditional passages, three novel forms are proposed, which are very hard to be manufactured by a common method. Furthermore, the geometrical features are selected optimally by means of full factorial experiments to balance the pressure loss and space requirement. Moreover, taking advantage of 3D printing, it is possible to build the passages in novel forms which are beyond the capacity of conventional manufacturing. Results show that the pressure loss can be reduced considerably by adopting a smooth transition, where the reduction can reach up to $50 \%$.
\end{abstract}

Keywords: hydraulic manifold; energy-saving; internal passages; 3D printing; pressure loss; computational fluid dynamic (CFD)

\section{Introduction}

Hydraulic manifolds, which house tortuous circuit layouts and are usually adopted in mobile machinery like mobile robots and civil machinery [1-4], can introduce high pressure loss. This is because the priority of minimizing mass and size outweighs the importance of reducing pressure loss. Whereas, the pressure loss affects the efficiency of the whole system. In fact, the energy efficiency of some hydraulic systems is only $\sim 22 \%[5,6]$. More critically, mobile hydraulic systems typically have an overall efficiency of approximately 14\% [7]. Nowadays, energy efficiency is becoming a key topic, arousing wide discussion among researchers and industry. Many investigations have been carried out to achieve the energy-saving design of hydraulic systems [8-14]. It is true that the main source of energy loss lies in valves, but the manifold with complex passages also accounts much for pressure drop [15] and the reduction of pressure loss in manifolds is also promising.

This problem of pressure loss in manifolds and relative scientific works are mainly found in the fluid machinery and hydraulic field. Early in the 1960s, the coefficients of local resistance and frictional loss in the piping system with distinct bend types were measured, and the empirical formulations were formed to estimate the pressure loss [16,17]. Even the wide deviations of loss coefficients are observed in various investigations, it gives us a hint that the abrupt change of flow direction can cause much energy consuming in a manifold [18]. Some experimental and theoretical analyses were given for turbulent flow in a bent pipe $[19,20]$. Usually, a single passage is a simple but useful object 
when analyzing a manifold. Zhang et al. optimized the duct nets in the hydraulic manifold using computational fluid dynamic (CFD) tools [21]. The separate flow perplexing eddies near the right angle intersection region is the key to energy loss, which is the bottleneck in the optimal design of the manifold. Geometry feature that destroys flow structures and causes fluid separation should be avoided. Moreover, the distance and orientation of the second elbow compared to the first one have a greater impact on the loss of energy. Furthermore, Song et al. studied the combination of bends in U-shape and S-shape [22]. The results show that the disturbance between two serial bends is weak if the distance is larger than five times diameter, and loss coefficient is no longer constant with the spanning angle ranging from $120^{\circ}$ to $140^{\circ}$. They testify the results applying the CFD analysis to a hydraulic manifold. Moreover, CFD is an effective tool and can be used to study the pressure drop along the passage with multiple elbows $[23,24]$. Whereas, the researches mentioned above circumscribe the optimization in traditional manufacturing methods.

To reduce pressure loss in manifolds, some possible solutions have been proposed. Actually, the casted channel was recommended to eliminate the sharp intersection in a manifold [21], but the minimum keyhole feature limits the wide application in the hydraulic manifold, and casting is not suitable for small-scale production. However, the situation has changed much since the emergence of $3 \mathrm{D}$ printing, which is also called additive manufacturing (AM) [25-28]. It provides unprecedented freedoms of design and makes it easy to realize complex and hollow shape forming. In the early stages, rapid casting (RC) was used to cast components with an intricate cavity which are either too expensive or impossible to be machined by conventional methods [29,30]. With the advancement of 3D printing, it has evolved into rapid manufacturing (RM), which can produce the functional part directly. Renishaw leverages the AM's ability to build passageways in a hydraulic manifold, with a mass reduction up to $79 \%$ and flow efficiency improvement up to $60 \%$ according to Renishaw Ltd [31]. Cooper et al. redesigned a manifold using direct metal laser sintering (DMLS) technology, which eliminates the rectangle bends in passages [32]. The experimental test shows that the flow velocity increases by $150 \%$ at $20 \mathrm{MPa}$ working pressure while weight is reduced. They prove the feasibility of $3 \mathrm{D}$ printing in manifolds, but energy efficiency was merely mentioned. Schmelzle et al. printed a 17-piece assembly as a single component using the powder bed fusion (PBF) technology [33]. They extracted the pipe net instead of drilling holes in the metal block. The final part is $60 \%$ lighter than the original one, but little fluid performance is addressed.

In order to obtain the manifolds causing less pressure drop, the novel design is presented. The single passages in manifolds are investigated first and then, three types of novel feature are proposed. The newly designed manifold is printed by a specific 3D printing technology and pressure test is carried out to testify the design concept. Also, some basic design rules that are applicable in AMed manifolds are given in this paper.

The remainder of this paper is organized as follows. In Section 2, typical passages in traditional manifolds are introduced, and some basic research is also performed. Section 3 gives detailed information about the simulation and experiment. Based on that, the detailed results are analyzed in Section 4. Further, the optimization and experimental validation are given in Section 5. Eventually, this paper is concluded in Section 6.

\section{Typical Passages in Manifolds}

Limited by the conventional manufacturing method, a traditional manifold is composed of straightly drilled and auxiliary holes. According to the fluid changes, the channel acts like confluence, fork, and direction guiding. Despite these different functions, the bend that formed by two ports on adjacent faces is a basic structure.

As shown in Figure 1, bends have some basic structures, like $90^{\circ}$ sharp elbow (I), expansion or contraction (II), and offset intersection (III). A manifold takes shape combining these structures in different orders and locations, so the investigation into a single structure can provide valuable information. 


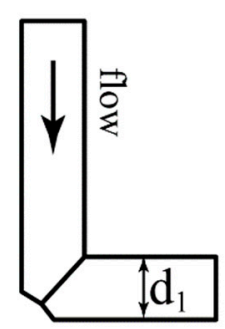

I-L

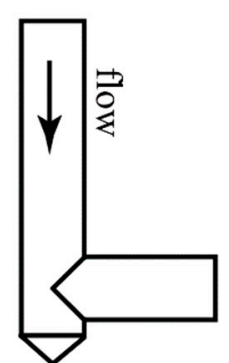

I-T

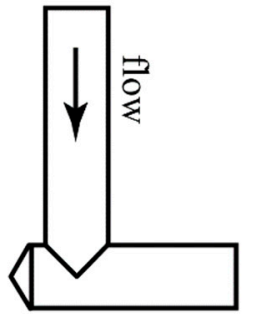

I-Ti

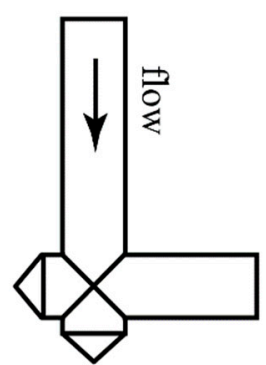

I-X

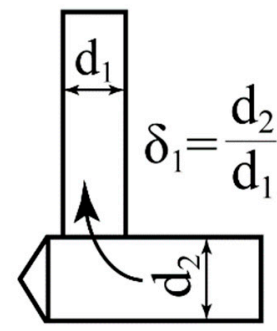

II- $\delta_{1} \mathrm{C}$

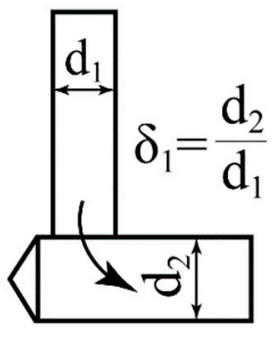

II- $\delta_{1} \mathrm{E}$

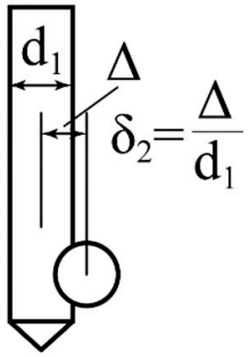

III- $\delta_{2}$

Figure 1. Typical passages in a manifold.

The $90^{\circ}$ sharp elbow (I) is the most common type in a manifold, which is formed by two in-plane holes drilled on the adjacent faces. It has two holes in the same diameters creating sharp $90^{\circ}$ corners. According to the amount of length that one hole has over another, Type I has a L-shaped, T-shaped, and X-shaped connection that is coded in I-L, I-T, and I-X respectively. Besides, the flow direction has effects on the pressure drop [34], so T-shaped connections will have another form when the flow direction is reversed, which is labeled using I-Ti.

Expansion/contraction (II) exists when two channels have different diameters. With a diameter of ports in either valves or measurement gauges fixed, the size of holes in a manifold is determined. If fluid enters the manifold in a smaller port and exits from a larger port, this case can be grouped as expansion. If the fluid flow from larger to smaller port, it can be grouped as contraction. Besides, the relative diameter of two holes is a determining factor that affects the flow patterns, so the diameter ratio $\delta_{1}$ is given to distinguish the geometry features. Here, passages with four different diameter ratios $\left(\delta_{1}=1,1.2,1.5,2\right)$ are investigated and are marked like II- $\delta_{1} C / E$, where $C$ means contraction and E means expansion.

It is challenging to choose proper flow paths to avoid the interference where enough clearance should be guaranteed while the volume of the manifold should be kept as small as possible. Under this circumstance, the offset intersection (III) is often a good choice. Offset distance $\Delta$ means how far there two holes are and relative offset distance $\delta_{2}$ is adopted to label the channel. In this research, we investigate four $\delta_{2}$ values-0, 0.2, 0.4, and 0.6-and they are denoted as III- $\delta_{2}$.

Considering the three types mentioned above, a sample containing nine single channels whose outlet diameters are $6 \mathrm{~mm}$, is designed to investigate the pressure drop, as illustrated in Figure 2. To ensure the accurate measurement of the pressure, the fully developed fluid is required at the inlet and the outlet. The fluid entry length before entering the bend is more than ten times over internal diameter $[35,36]$. Also, two pressure transducers are double diameter away from the inlet and outlet. The pressure loss is obtained by calculating $\mathrm{P}_{\text {inlet }}-\mathrm{P}_{\text {outlet }}$. 


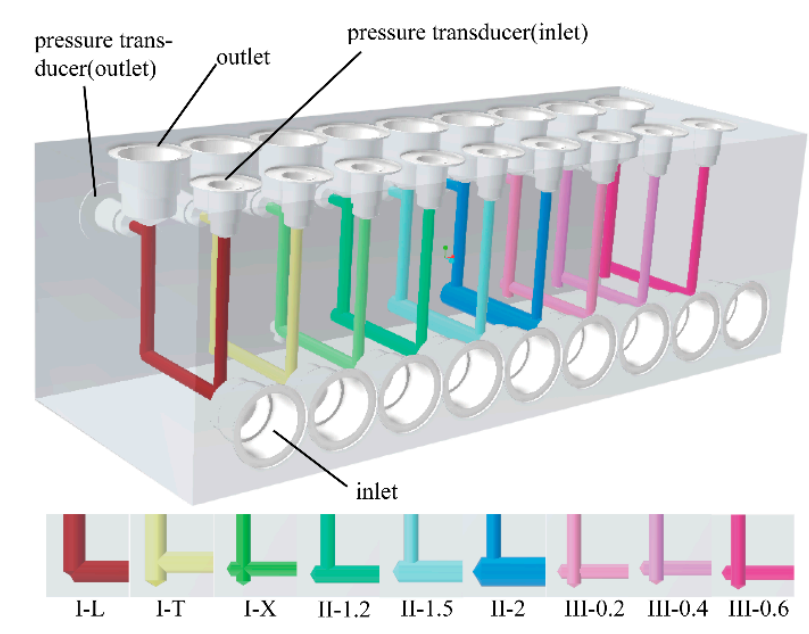

Figure 2. A manifold sample containing nine single passages.

\section{Simulation and Experiment Details}

\subsection{CFD Simulation Settings}

CFD is a useful tool validating the ideas and can provide valuable references in the design process, and Fluent, a commercial CFD software, is adopted in this research. Before continuing, some basic assumptions should be made:

1. The fluid properties of the working fluid keep unchanged and no phase changes happen in the passage.

2. Physical properties such as density and viscosity of the working fluid are evenly distributed and isotropic.

3. This simulation is not a solid-liquid coupling analysis system and the interaction forces are negligible.

4. The entire system under consideration is isothermal.

5. The flow is assumed to be incompressible.

In this research, the ISO VG 46 mineral oil has been used as the working fluid, whose density is $860 \mathrm{~kg} / \mathrm{m}^{3}$ and dynamic viscosity is $0.03956 \mathrm{~Pa} \cdot \mathrm{s}$ at an ambient temperature about $40^{\circ} \mathrm{C}$. The flow rate of the inlet varies in the range of 5 to $25 \mathrm{~L} / \mathrm{min}$. At the outlet, the boundary condition is set as pressure-out. Although the Reynolds number (400-1956) in this paper is smaller than the critical one, the turbulent model is still suitable because of the acute change of fluid in the bend. The RNG k- $\varepsilon$ model is chosen and the discretized equations are solved by the SIMPLE algorithm. In spatial discretization, the second-order schema is applied for momentum, the first-order upwind schema for momentum, and second-order upwind schema for both turbulent kinetic energy and turbulent dissipation rate. The under-relaxation parameters are set to 0.3 for pressure, 1 for density and body forces, 0.7 for the momentum terms, 0.8 for both turbulent kinetic energy and turbulent dissipation rate, and 1 for turbulent viscosity. Additional boundary conditions are also important to the simulation [36]. Usually, the turbulent kinetic energy $\mathrm{k}$ and the turbulent energy dissipation $\varepsilon$ are obtained from experimental data or empirical formulas. For fully developed fluid, $\mathrm{k}$ and $\varepsilon$ can be calculated by giving turbulent intensity and hydraulic diameter. The turbulent intensity is calculated using Equation (1). Substituting $\mathrm{Re}_{\mathrm{DH}}$ with the Reynolds number above, the result ranges from $6.2 \%$ to $7.5 \%$ and $6.5 \%$ is set in the simulation. The hydraulic diameter is the same as the diameter of the inlet port for pipes.

$$
I=0.16\left(\operatorname{Re}_{\mathrm{DH}}\right)^{-1 / 8}
$$

Mesh sensitivity analysis is performed to ensure that the result is independent of the number of nodes, and it is a necessary trade-off between the accuracy and computational costs. Near the passage 
wall, the fluid states are quite complex and the element size should be fine enough to make sure that nodes can contain the information of the viscous sublayer accurately. Therefore, the standard wall function is applied to the passage wall which is specified as stationary and no-slip. Mesh ratio [15]the maximum element size divided by the minimum channel diameter, increases from 0.05 to $0.2-$ and the corresponding result are presented in Figure 3. When the mesh ratio decreases, the number of nodes increases from 25,317 to 480,524. As illustrated in the figure, the improvement in pressure drop caused by finer mesh is weakened gradually and the fitting curve also gives the same trend, so 0.07 is finally employed. Besides, the detailed information about the nodes in the different simulation case is given in Appendix A.

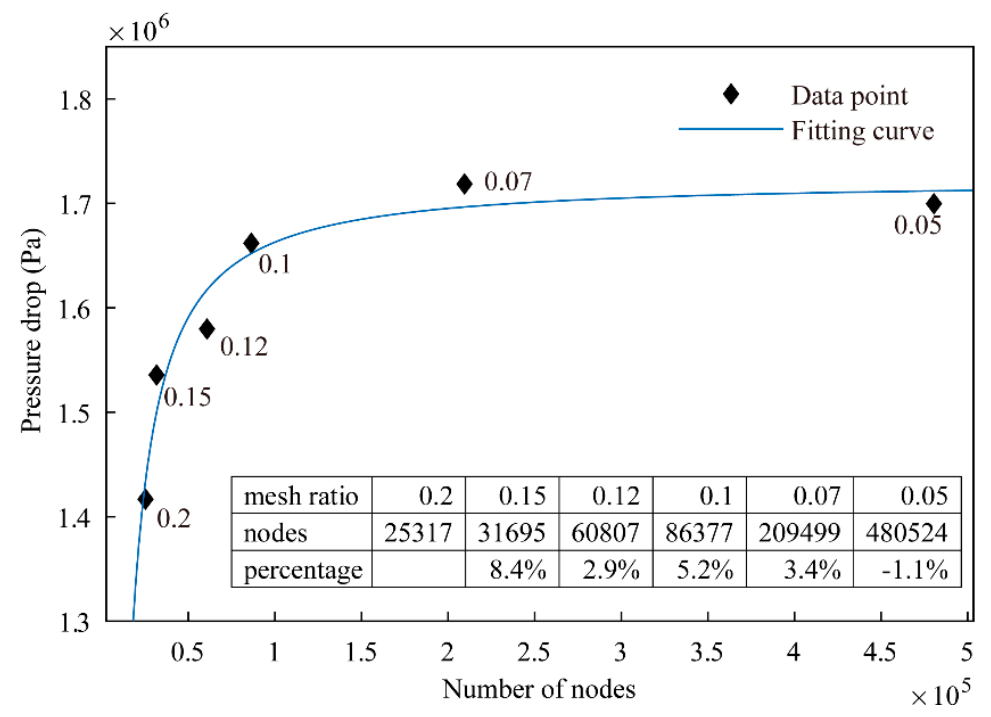

Figure 3. Sensitivity of mesh.

\subsection{Test Rig}

To verify the reliability of CFD, simulation results are compared to the data measured from the sample manifold. The schema of the test rig is illustrated in Figures 4 and 5. The main parts are described below.

1. The power unit integrating a fixed displacement pump and a $5.5 \mathrm{~kW}$ electric motor.

2. The safety device, mainly the relief valves. Relief valve 7 has the highest pressure to ensure the testing pressure in the safety range.

3. The manifold and measuring gauges. The manifold is fixed on the test bed and is connected to the $\mathrm{P}$ and $\mathrm{T}$ ports. The measuring instruments are two pressure transducers 4 and 5 and a flow meter 6 , and the data are recorded and processed using a computer. The data acquisition devices along with corresponding characteristics are given in Table 1.

To take accurate measurements as much as possible, the fluid should travel some distance to develop fully before entering the manifold [35]. Discussed by Zardin et al. [15], the distance between connectors should be kept over 13d, where $d$ is the inner diameter of the channel. At this distance, the fluid will be developed fully and won't be disturbed by each other. The final results are obtained by feeding flow rate at $25 \mathrm{~L} / \mathrm{min}$.

Table 1. Characteristics of measuring device.

\begin{tabular}{ccc}
\hline Sensor/Data Acquisition & Range & Accuracy \\
\hline piezoresistive transducer & $0-160 \mathrm{bar}$ & $<0.5 \% \mathrm{fs}$ \\
Turbine Flow Meter & up to $116 \mathrm{~L} / \mathrm{min}$ & $<0.5 \% \mathrm{fs}$ \\
PCI 6211 & $\pm 10.4 \mathrm{~V}$ & \\
\hline
\end{tabular}




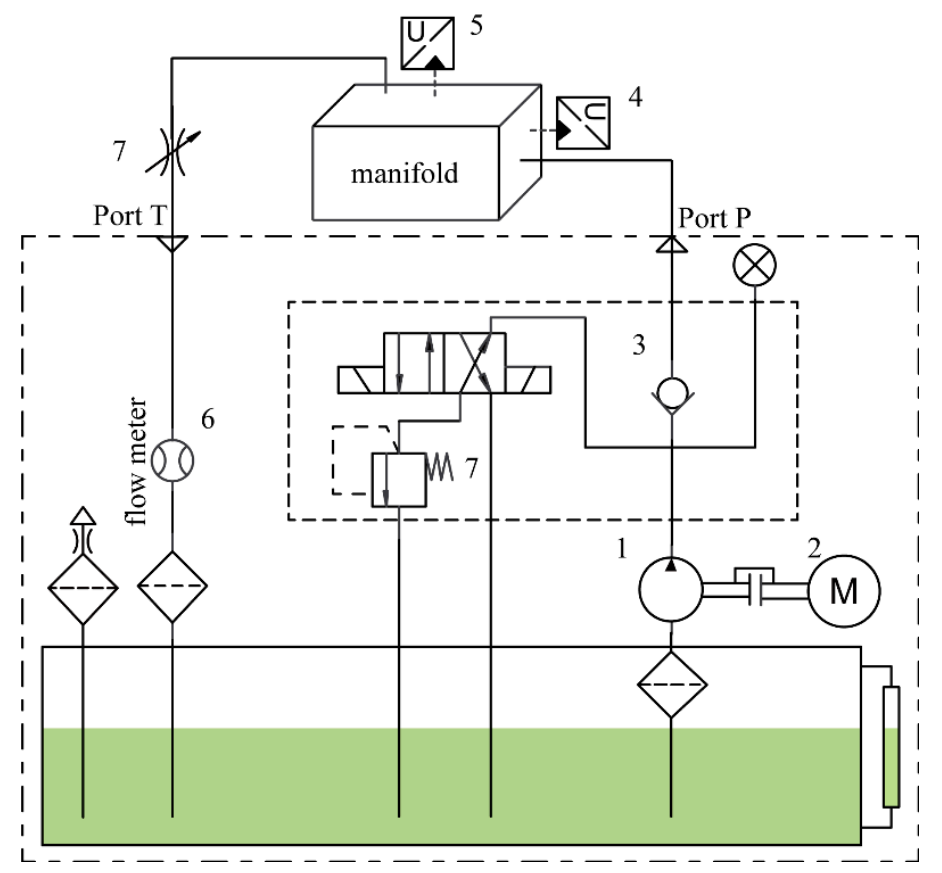

Figure 4. Schema of the test rig: 1-hydraulic pump; 2-motor; 3-check valve; 4,5-pressure transducer; 6-flow meter; 7-throttle valve.

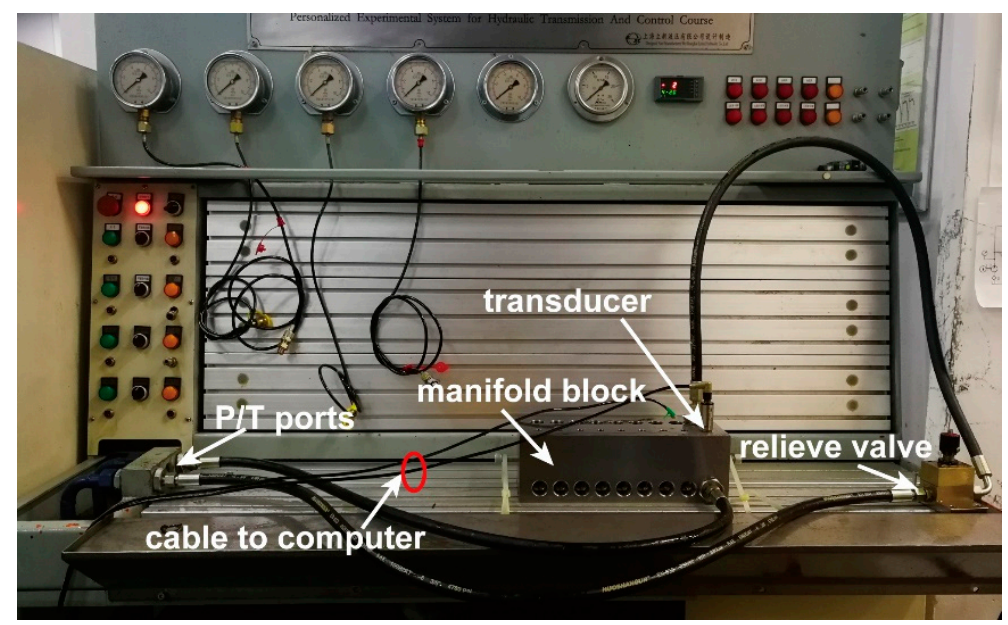

Figure 5. Test rig and connections.

\section{Results and Discussion}

Thirteen data points are recorded in Figure 6 from both simulation and experiment. It is obvious that type III has a much larger pressure drop compared to the others whether in the simulation or experiment, and this may ascribe to the acute disturbance in the offset intersection region. By contrast, II-1.5E and II-2E have a lower pressure drop and different flow direction, which leads to much difference when comparing contraction with expansion in type II. The largest difference can reach up to about 1.3 bar in III-0.6, leaving a gap about $20 \%$ between two methods. This may attribute to the two main reasons:

1. Seen in Figure 2, we set the ports for pressure transducers near to inlet or outlet as much as possible, but the real length is shorter than that in simulation.

2. Auxiliary holes drilled for sensors are not in the same length, and the longer holes in inlet may cause the gap between simulation and experiment. 
However, the trends of the pressure drop correspond well to each other, so the simulation model is good enough to carry out further researches.

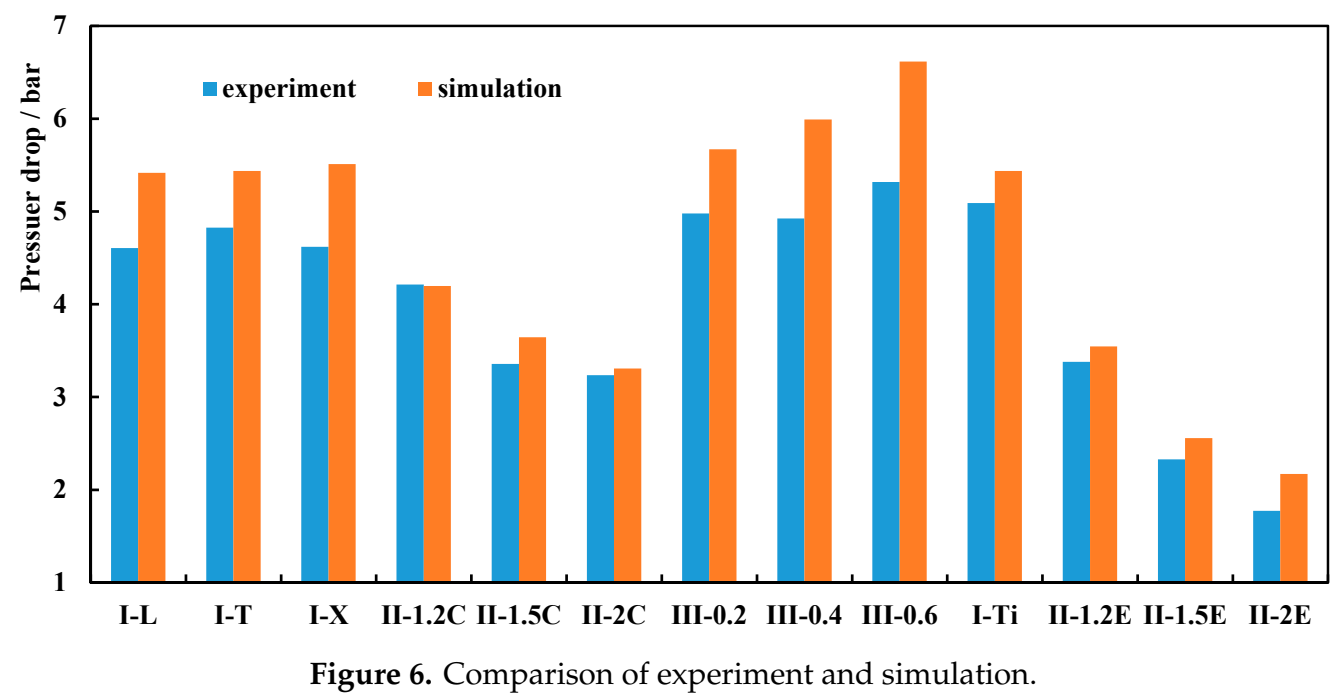

\section{1. $90^{\circ}$ Sharp Elbow (I)}

Pressure and velocity information was extracted, and Figure 7 shows the pressure distribution on the symmetry plane as well as the velocity vector in the local region. Extracted along the central axis as marked in dash line in Figure 7a, 2000 points containing pressure and velocity information are depicted in Figure 8. Generally speaking, the pressure drop in a passage consists of two parts, namely local pressure loss and frictional loss along the channel walls.

Along the inlet pipe, the pressure goes down slowly which can be seen both in cloud map and data chart, and this is the effect of friction along the wall. However, at the same time, the gradually stabilized velocity curve means the fluid develops fully before entering the bend. Because of an abrupt stop at the bend and inertia of the fluid, the fluid is jetted on the wall downstream, which, thus, causes a high-pressure region near the wall. At this region, the pressure and velocity change sharply and can be read in Figure 8. The velocity curve has high and low peak values, this is because the central axis crosses the mainstream that has nonuniform velocity distribution on the cross section after leaving the bend, which is also called the secondary flow [37-39]. After discharging, the velocity increases gradually and stops at a stable value, also the pressure distribution along the outlet become uniform after leaving the bend. This means the fluid goes back to normal.

The pressure drops $\Delta \mathrm{P}_{1}$ in the Table 2 is the difference between face-averaged pressure on the inlet and outlet. It shows that I-X has the highest pressure drop while the I-L has the lowest pressure drop. This can be ascribed to two reasons: (1) the vortex formed in the cavity causes energy consuming and (2) the accelerated fluid downstream increases the frictional loss. 

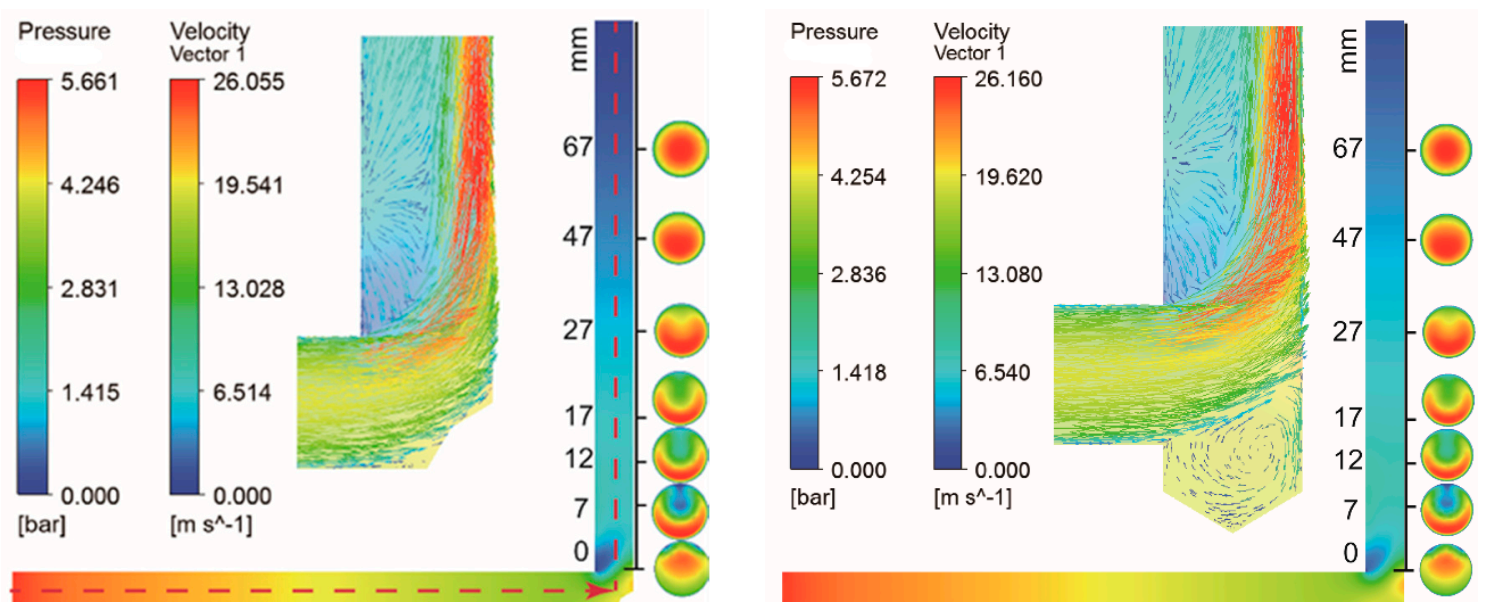

(a)

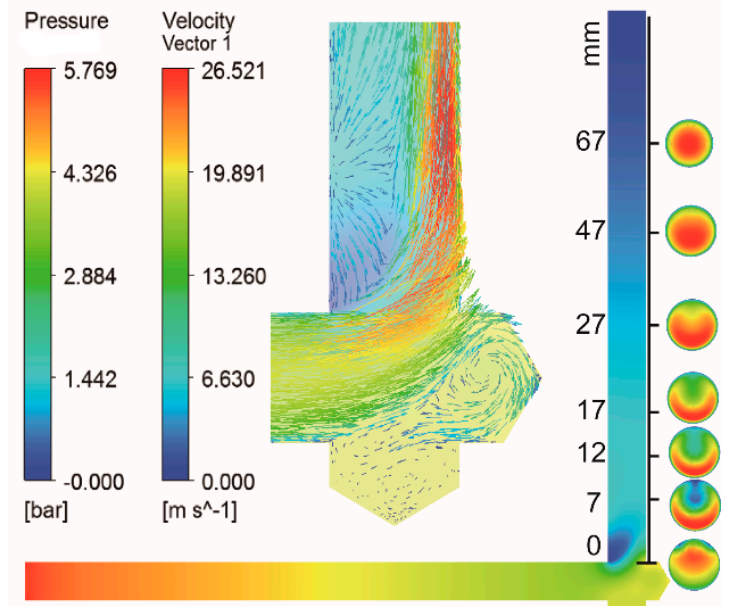

(b)

(c)

Figure 7. Pressure and velocity distribution in (a) I-L, (b) I-T, and (c) I-X passage.

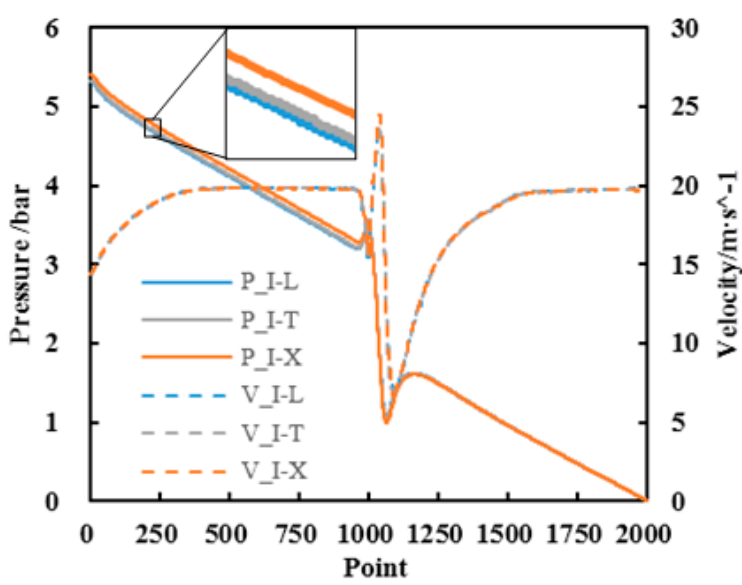

Figure 8. Pressure and velocity along passages I.

Table 2. Pressure drop in the passage I.

\begin{tabular}{ccccc}
\hline Type & I-L & I-T & I-Ti & I-X \\
\hline$\Delta \mathrm{P}_{1}$ (bar) & 5.415 & 5.436 & 5.435 & 5.511 \\
\hline
\end{tabular}


The detailed view of the velocity vector in elbows denotes the occurrence of local circulation. Compared with I-T and I-X, the I-L has no cavity formed by excessive length, so there is no space for vortex development. In I-T and I-X, the cavity is filled with vortexes that caused by the tangential velocity of the fluid. Using velocity and pressure information on the points, Figure 8 shows the pressure difference marked in the black square. This can be ascribed to the energy consumption of the vortex in the bend.

Overall, the pressure loss in channels with the sharp bend is quite large and a more smooth transition is needed to reduce the pressure decline.

\subsection{Contraction/Expansion (II)}

Compared with in-plane bends, the intersection with two different diameters shows quite different fluid patterns. The contraction and expansion channels are in the T form, so I-T whose $\delta_{1}$ equals 1 is chosen as the reference. The pressure drop in Table 3 decrease when $\delta_{1}$ increase from 1 to 2 in the bidirectional flow. This means the larger $\delta_{1}$, the smaller pressure drop is under a fixed flow rate. From Figure 9, the evenly distributed pressure and velocity are observed in the passage with a larger diameter under both contraction and expansion conditions. The evenly distributed velocity and pressure are related to a relatively stable fluid state in the bend region. Also, the peak value of velocity is not so high, this is because a larger diameter makes a large space for the fluid and can absorb much turbulence.

Comparing Figure 9a with Figure $7 \mathrm{~b}$, there is no vortex and the secondary flow is quite weak downstream. Different from jetting on the wall downstream, the fluid is squeezed from a high-pressure chamber to the outlet. In Figure 9b, even the vortexes exist in cavity and downstream, it locates in the low-pressure region which may be free from much energy dissipation. Thereafter, the pressure drop is reduced due to the reduction of the velocity and the vortex region is much smaller compared to the I-T. Thereafter, the pressure drop is reduced due to the reduction of the velocity and the vortex region is much smaller compared to the reference one.

From Figure 10, the effect that velocity has on the friction loss is obvious. Different values of $\delta_{1}$ cause different inlet velocity at the same flow rate under expansion or contraction condition. In the contraction, the pressure drop before entering the bend is proportional to the velocity, but the pressure under three flow rate is quite the same after leaving bend. However, the expanded one gives a different result. A constant deviation along the inlet port is observed. The deviation $a$ and $b$ is stable before the bend, while downstream pressure loss a' and b' has the same trend observed in the inlet pipe in contraction. This means that the velocity accounts much fot friction loss while bend takes effect locally. However, the negative pressure region can be seen in figure and chart, so the value of $\delta_{2}$ should be chosen carefully.

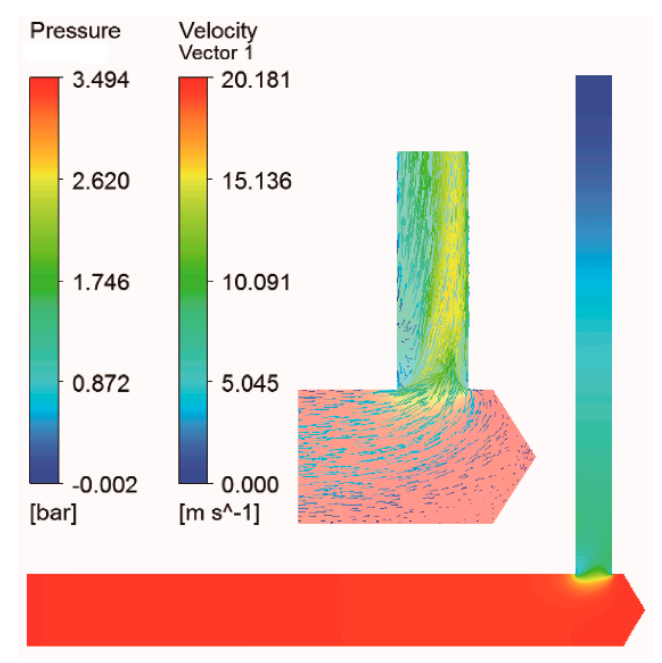

(a)

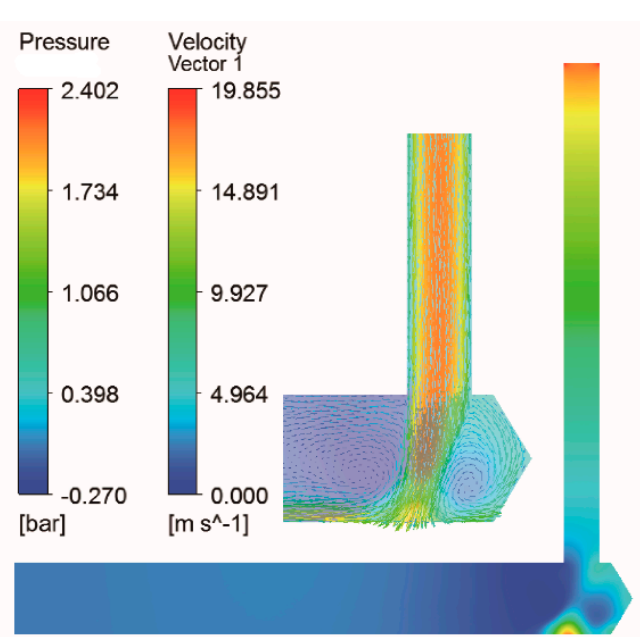

(b)

Figure 9. Pressure and velocity distribution when $\delta_{1}=2$ : (a) contraction and (b) expansion. 


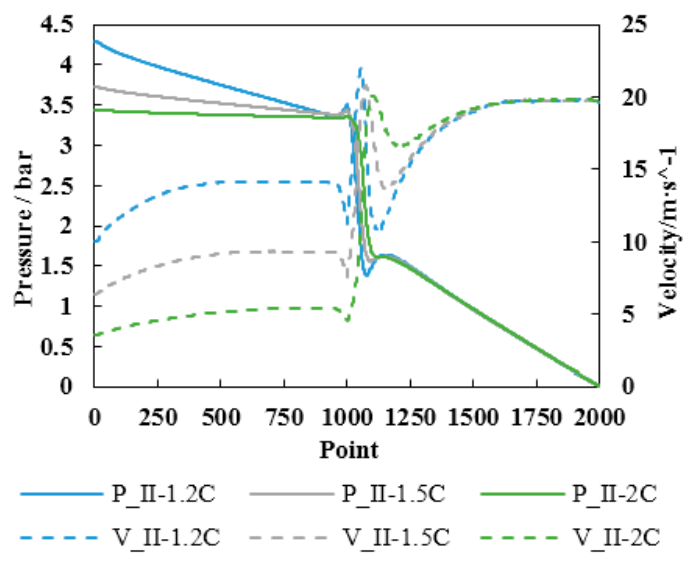

(a)

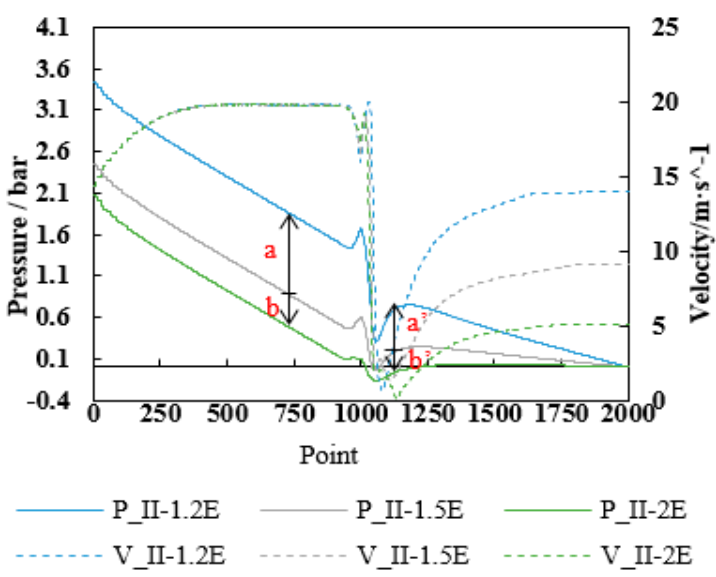

(b)

Figure 10. Pressure and velocity along passages II: (a) contraction and (b) expansion.

Table 3. Pressure drop in contraction/expansion.

\begin{tabular}{ccccc}
\hline Type & I-T & II-1.2 & II-1.5 & II-2 \\
\hline Contraction (bar) & 5.436 & 4.195 & 3.643 & 3.307 \\
Expansion (bar) & 5.436 & 3.545 & 2.557 & 2.170 \\
\hline
\end{tabular}

\subsection{Offset Intersection (III)}

Type III has X-shaped intersection form but with offset, so the pressure drop in the I-X is given as the reference where $\delta_{2}$ equals 0 . Table 4 shows that the pressure drop increases when offset distance ranges from 0 to $3.6 \mathrm{~mm}$.

Shown in Figure 11, the maximum velocity is larger than any other channels, this is because the effective flow area is reduced with $\delta_{2}$ increasing. As a result, the velocity of the fluid through the bend is prompted under a constant flow rate, which will cause large frictional loss downstream as discussed above. Besides the increase of the velocity, the distribution downstream differs much compared to other channels. We can discern the rotation movement of the fluid through the mainstream region denoted in the circular cloud map along the outlet pipe. In Figure 12, the vortex is formed downstream, but this only causes small difference which can be read in Figure 11. For offset intersection, the pressure loss deviation is mainly the result of the abrupt change of the flow area in the bend. Under this circumstance, the stable flow area around the bend may contribute to a smaller pressure drop.

Table 4. Pressure drop in offset intersection.

\begin{tabular}{ccccc}
\hline Type & I-X & III-0.2 & III-0.4 & III-0.6 \\
\hline$\Delta \mathrm{P}_{2}$ (bar) & 5.511 & 5.670 & 5.991 & 6.616 \\
\hline
\end{tabular}



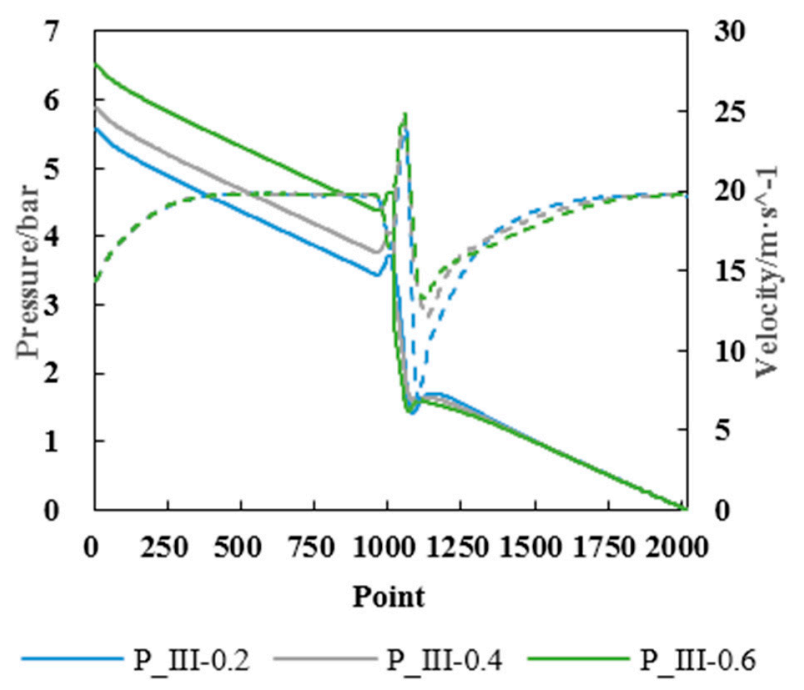

- - - - V_III-0.2 - - - - V_III-0.4 - - - - V_III-0.6

Figure 11. Pressure and velocity along passages III.

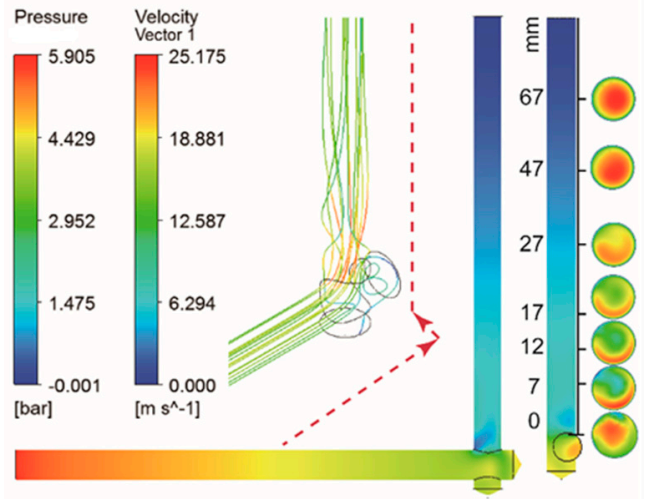

(a)

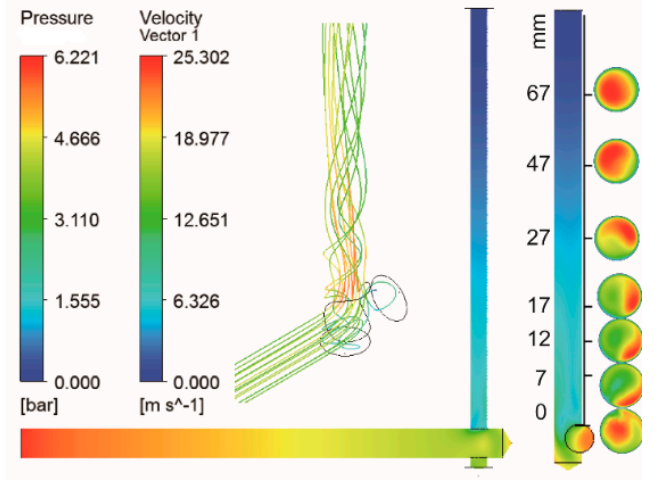

(b)

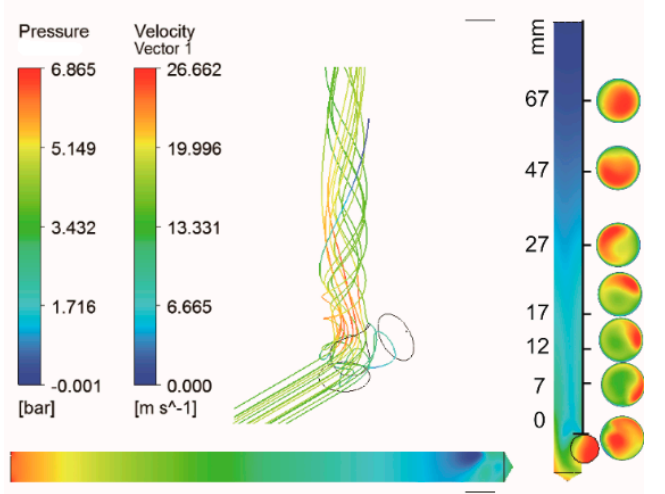

(c)

Figure 12. Pressure and velocity distribution: (a) III-0.2, (b) III-0.4, and (c) III-0.6.

\section{Structure Optimization and Validation}

As discussed above, the abrupt change of the flow direction causes much local pressure loss and frictional loss near the corners, so eliminating the sharp transition is an effective way to reduce local pressure drop. 


\subsection{Design of Novel Passages}

Shown in Figure 13, the sharp intersection is replaced with a smooth arc transition, and this is realized by adding round corners to passages. In order to provide general understandings and give some basic rules, dimensionless feature sizes were adopted. The newly designed passage is labeled as C- $r_{\mathrm{i}} / r_{\mathrm{o}}$, where $r_{\mathrm{i}}$ or $r_{\mathrm{O}}$ is the feature size of the inner or outer corners, respectively. Actually, the feature size can be very large without any limitation, so some other factors should be taken into consideration. As illustrated in Figure 13, the area of bend region that is highlighted in a red rectangle and the bend volume are selected as additional indicators. They are denoted as $S_{\mathrm{C}} / S_{\mathrm{L}} / S_{\mathrm{X}}$ and $V_{\mathrm{C}} / V_{\mathrm{L}} / V_{\mathrm{X}}$ respectively, where $\mathrm{C}, \mathrm{L}$, or $\mathrm{X}$ presents a specific channel. Similarly, $\Delta \mathrm{P}_{\mathrm{CL}}$ and $\Delta \mathrm{P}_{\mathrm{CX}}$ are the pressure drops between two channels. Though the complexity of space allocation in a manifold is beyond two factors, these two may provide some useful information when designing a manifold hopefully. Noteworthy, $r_{\mathrm{i}}$ and $r_{\mathrm{o}}$ change independently, and this means it is supposed to select the optimal diameters for inner and outer corners in C- $r_{\mathrm{i}} / r_{\mathrm{O}}$.
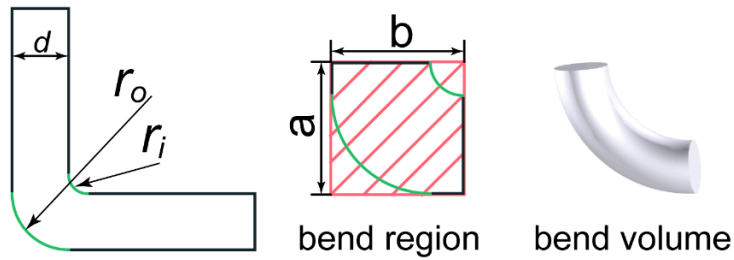

Figure 13. Design for a smooth transition $C-r_{\mathrm{i}} / r_{\mathrm{o}}$.

It is obvious that the novel passage degrades into L-shaped channel when $r_{\mathrm{i}}$ or $r_{\mathrm{o}}$ equals zero, but for convenience in analyzing, the pressure drops in I-L and I-X are both provided as references. The radii $r_{\mathrm{i}}$ and $r_{\mathrm{o}}$ were selected from $\{1.2,2.4,3.6,4.8,6\}$ and the full factorial design was performed. The simulation was performed under the flow rate of $15 \mathrm{~L} / \mathrm{min}$ and results are listed in Table 5 . The ratio between pressure reduction and bend area or bend volume is calculated to measure the space cost using a novel design. Compared to conventional channels, the newly designed ones occupy much more space in most cases. Generally, the spaces requirement increases along with the growth of inner and outer corner dimensions.

Table 5. Results of full factorial design for $C-r_{i} / r_{o}$.

\begin{tabular}{ccccccccc}
\hline $\begin{array}{c}\text { Channel } \\
\text { Type }\end{array}$ & $\frac{S_{C}}{S_{L}}$ & $\frac{S_{C}}{S_{X}}$ & $\frac{\Delta P_{C L}}{S_{L}}$ & $\frac{\Delta P_{C X}}{S_{C}}$ & $\frac{V_{C}}{V_{L}}$ & $\frac{V_{C}}{V_{X}}$ & $\frac{\Delta P_{C L}}{V_{C}}$ & $\frac{\Delta P_{C X}}{\frac{V_{C}}{V_{X}}}$ \\
\hline $\mathrm{C}-1.2 / 1.2$ & 1.44 & 0.5398 & 0.7085 & 1.9335 & 1.5171 & 1.1344 & 0.6725 & 0.92 \\
$\mathrm{C}-1.2 / 2.4$ & 1.44 & 0.5398 & 0.7184 & 1.9606 & 1.487 & 1.1119 & 0.6957 & 0.9518 \\
$\mathrm{C}-1.2 / 3.6$ & 1.44 & 0.5398 & 0.7153 & 1.9519 & 1.4686 & 1.0981 & 0.7013 & 0.9595 \\
$\mathrm{C}-1.2 / 4.8$ & 1.44 & 0.5398 & 0.7207 & 1.9668 & 1.4465 & 1.0816 & 0.7175 & 0.9815 \\
$\mathrm{C}-1.2 / 6$ & 1.44 & 0.5398 & 0.719 & 1.9623 & 1.6139 & 1.2067 & 0.6416 & 0.8777 \\
$\mathrm{C}-2.4 / 1.2$ & 1.96 & 0.7347 & 0.5369 & 1.4653 & 1.7768 & 1.3285 & 0.5923 & 0.8103 \\
$\mathrm{C}-2.4 / 2.4$ & 1.96 & 0.7347 & 0.5328 & 1.4539 & 1.7783 & 1.3297 & 0.5872 & 0.8033 \\
$\mathrm{C}-2.4 / 3.6$ & 1.96 & 0.7347 & 0.5416 & 1.4781 & 1.7613 & 1.317 & 0.6027 & 0.8246 \\
$\mathrm{C}-2.4 / 4.8$ & 1.96 & 0.7347 & 0.5391 & 1.4713 & 1.7242 & 1.2892 & 0.6129 & 0.8384 \\
$\mathrm{C}-2.4 / 6$ & 1.96 & 0.7347 & 0.5446 & 1.4863 & 1.6651 & 1.245 & 0.6411 & 0.8771 \\
$\mathrm{C}-3.6 / 1.2$ & 2.56 & 0.9596 & 0.4158 & 1.1348 & 2.0859 & 1.5597 & 0.5103 & 0.6982 \\
$\mathrm{C}-3.6 / 2.4$ & 2.56 & 0.9596 & 0.4167 & 1.1372 & 2.0542 & 1.5359 & 0.5194 & 0.7105 \\
$\mathrm{C}-3.6 / 3.6$ & 2.56 & 0.9596 & 0.4201 & 1.1464 & 2.059 & 1.5395 & 0.5223 & 0.7146 \\
$\mathrm{C}-3.6 / 4.8$ & 2.56 & 0.9596 & 0.421 & 1.149 & 2.0428 & 1.5275 & 0.5276 & 0.7218 \\
$\mathrm{C}-3.6 / 6$ & 2.56 & 0.9596 & 0.4168 & 1.1375 & 1.9421 & 1.4521 & 0.5495 & 0.7517 \\
$\mathrm{C}-4.8 / 1.2$ & 3.24 & 1.2145 & 0.3342 & 0.912 & 2.4091 & 1.8014 & 0.4495 & 0.6149 \\
$\mathrm{C}-4.8 / 2.4$ & 3.24 & 1.2145 & 0.3364 & 0.9181 & 2.3572 & 1.7625 & 0.4624 & 0.6326 \\
$\mathrm{C}-4.8 / 3.6$ & 3.24 & 1.2145 & 0.3344 & 0.9125 & 2.3139 & 1.7301 & 0.4682 & 0.6406 \\
$\mathrm{C}-4.8 / 4.8$ & 3.24 & 1.2145 & 0.3372 & 0.9202 & 2.3201 & 1.7348 & 0.4709 & 0.6443 \\
$\mathrm{C}-4.8 / 6$ & 3.24 & 1.2145 & 0.3352 & 0.9147 & 2.2624 & 1.6917 & 0.48 & 0.6567 \\
$\mathrm{C}-6 / 1.2$ & 4 & 1.4994 & 0.2725 & 0.7435 & 2.6732 & 1.9988 & 0.4077 & 0.5578 \\
$\mathrm{C}-6 / 2.4$ & 4 & 1.4994 & 0.2745 & 0.749 & 2.6228 & 1.9612 & 0.4186 & 0.5726 \\
$\mathrm{C}-6 / 3.6$ & 4 & 1.4994 & 0.276 & 0.7531 & 2.5931 & 1.9389 & 0.4257 & 0.5823 \\
$\mathrm{C}-6 / 4.8$ & 4 & 1.4994 & 0.2743 & 0.7485 & 2.6014 & 1.9452 & 0.4217 & 0.577 \\
$\mathrm{C}-6 / 6$ & 4 & 1.4994 & 0.2751 & 0.7508 & 2.5956 & 1.9408 & 0.424 & 0.58 \\
\hline & & & & & & & & \\
\hline
\end{tabular}


In Figure 14, enhancement, defined by dividing pressure drop $\Delta \mathrm{P}_{\mathrm{L}}$ and $\Delta \mathrm{P}_{\mathrm{X}}$ in $\mathrm{L}$ - and $\mathrm{X}$-shaped channels by $\Delta \mathrm{P}_{\mathrm{C}}$ for novel channels, is larger than 1 . This means the new design can reduce pressure drop in all cases. By contrast to conventional channels, the pressure reduction can reach up to 0.25 bar, which means the energy efficiency can be raised by $\sim 10 \%$. However, the improvement taking bend region and bend volume into consideration is not very satisfying. As indicated in Figure 15, the reduction of pressure drop over the bend region and channel volume is reduced. The downward tendency does not mean there is no improvement, but the 'margin effect' that efficiency enhancement/space requirement is reduced and the space requirement is becoming more important when adopting arc transition. Besides, there is always a gap between L- and X-related points. That can be explained by the fact that with the almost same pressure drop in two channels, a larger area and volume of bend in X-shaped channel limits the performance.

Further, we expanded the range of $r_{\mathrm{i}}$ or $r_{\mathrm{o}}$ to $[0,18]$, and the similar results are observed but with sharper reduction for efficiency enhancement/space requirement as given in Figure 16 . When $r_{\mathrm{i}}$ approaches 12, improvements are reduced to a lower level compared to other results. This means that choosing a larger feature size will not raise 'margin effect'. Also, some peak values are observed in Figures 16 and 17. The occurrence of peak value is consistent with C-*/18, which may be attributed to the reduced effective flow area. With the inner corner fixed, the larger feature size of the outer corner, the smaller effective flow area becomes, thus leads to the pressure drop increases. So, the moderate effective flow area should be guaranteed when using a smooth transition.

Based on the analysis above, the final design was C-6/9. With this parameter pair, a smoother flow area than conventional ones and a better improvement over bend region and channel volume can be obtained.

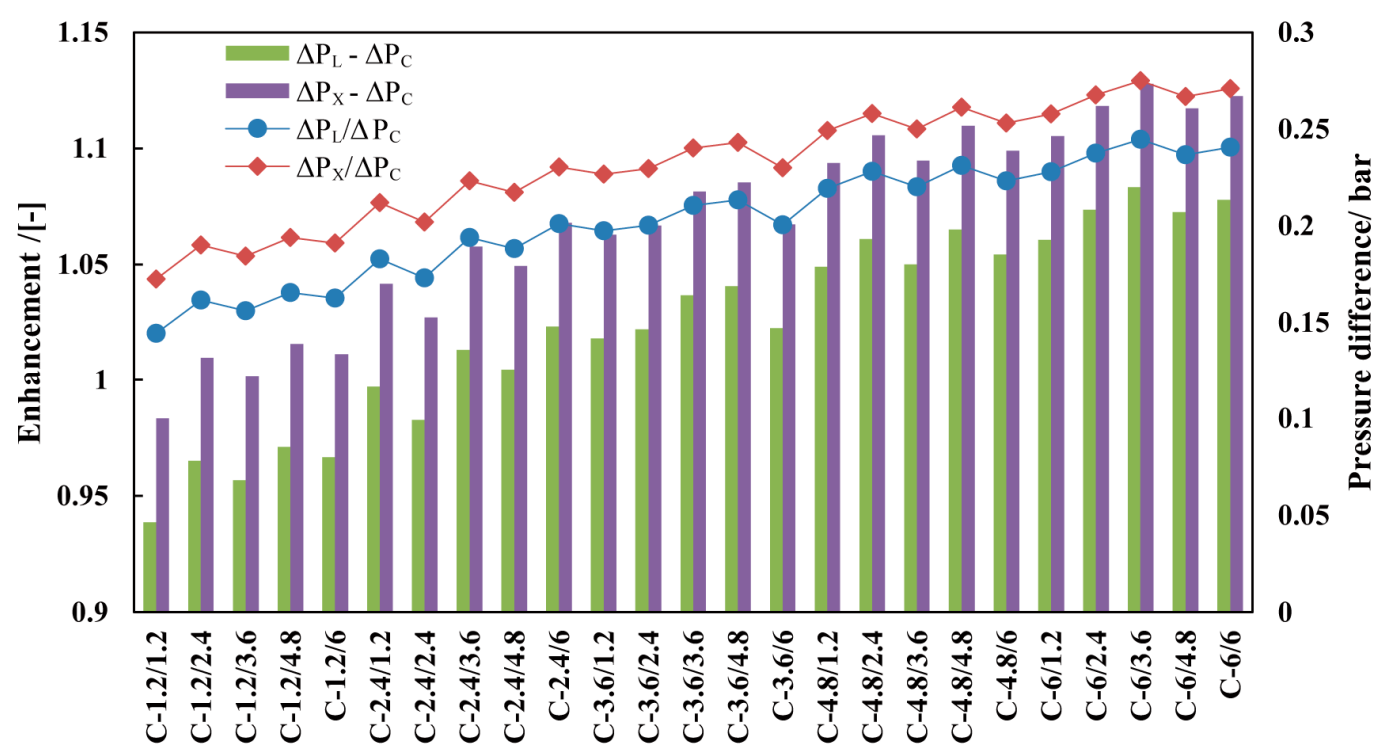

Figure 14. Improvement for C- $r_{\mathrm{i}} / r_{\mathrm{o}}$ compared to I-X and I-L $(0.2 \leq r \leq 1)$. 


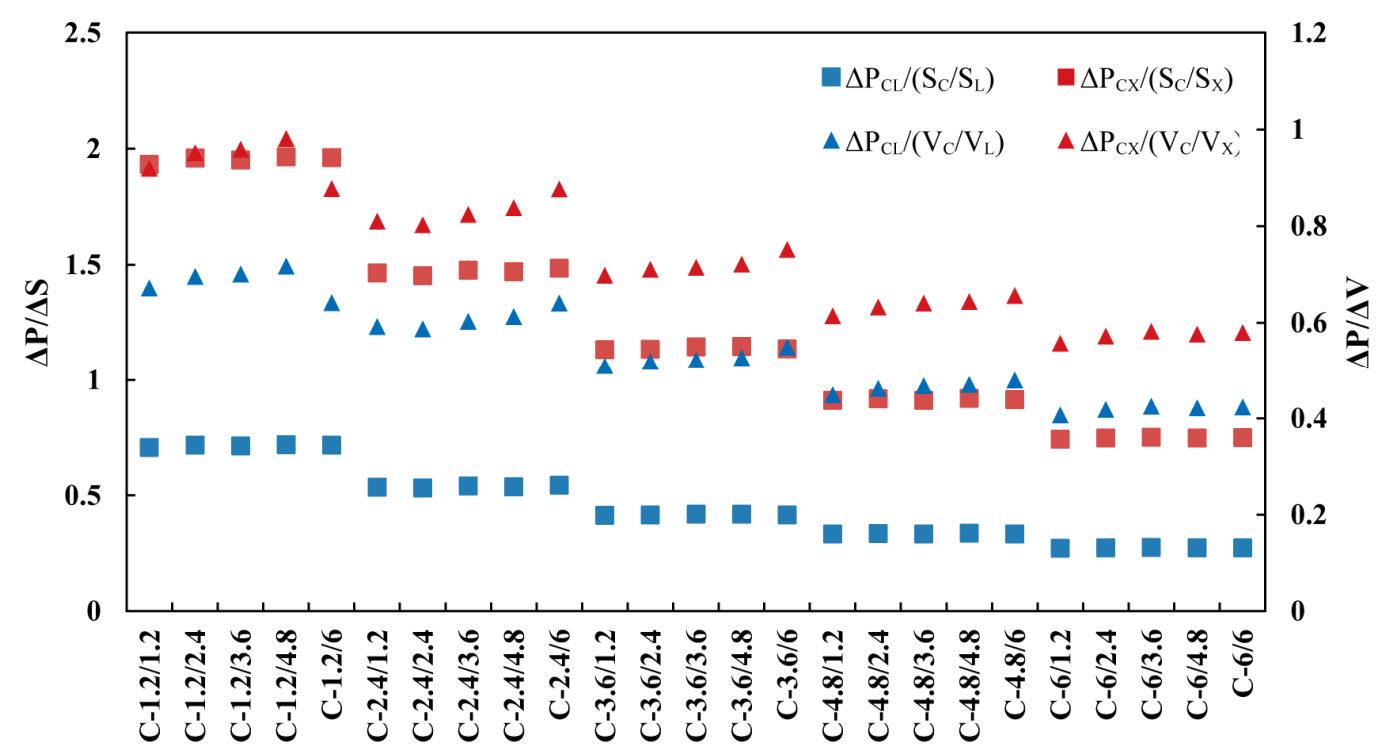

Figure 15. Improvement over bend region and bend volume $(1.2 \leq r \leq 6)$.

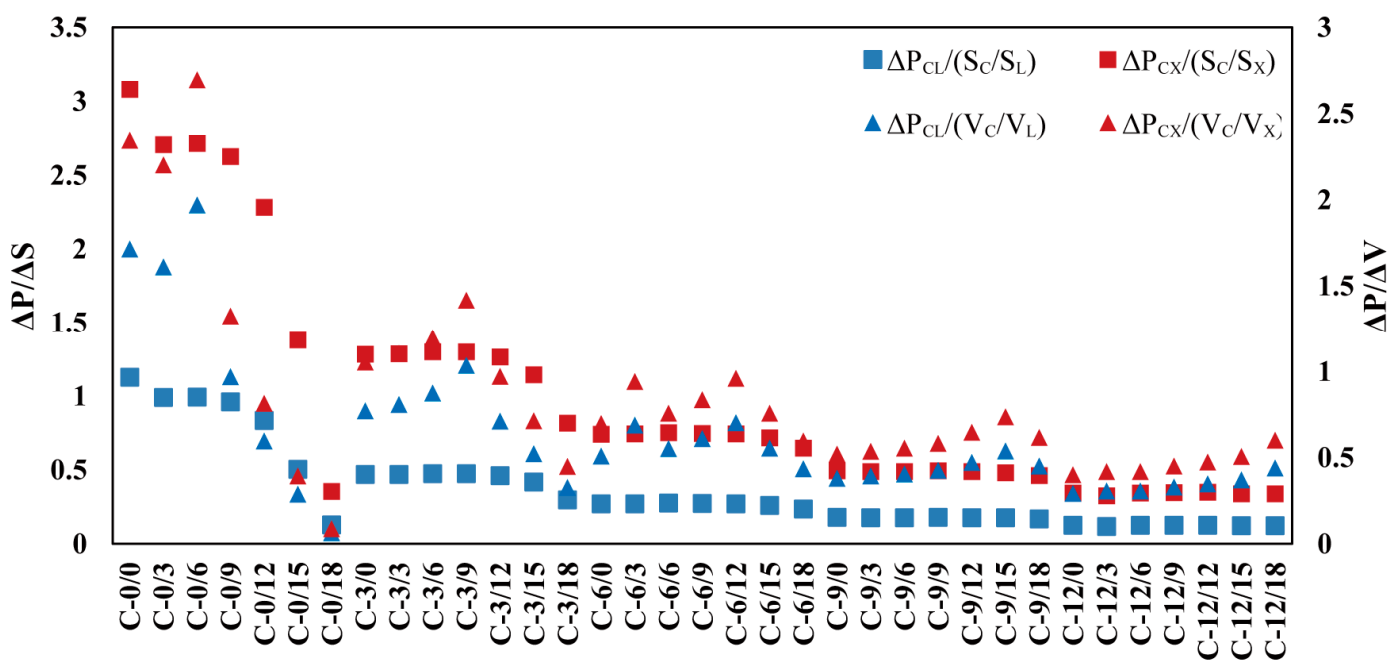

Figure 16. Improvement over bend region and bend volume $(0 \leq r \leq 18)$.

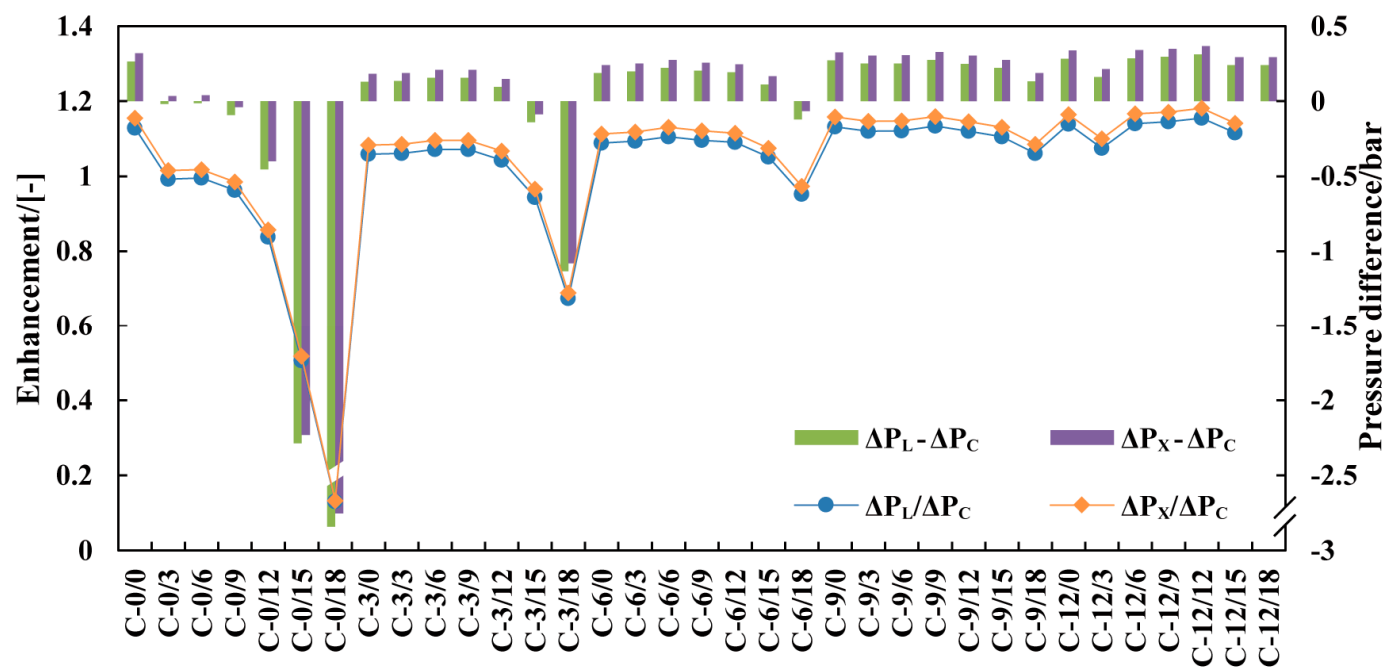

Figure 17. Improvement for C- $r_{\mathrm{i}} / r_{\mathrm{o}}$ compared to I-X and I-L $(0 \leq r \leq 18)$. 
For the passage II, the loft feature can be applied to three local regions as shown in Figure 18, and is denoted as Lo-i, -ii, and -iii. If we design a lofted passage, there are eight choices but without knowing the exact performance. So a design containing three factors was designed, and the simulation environment was the same as that in type I. Similarly, the pressure drop under expansion and contraction condition was recorded. The result marked in red in Table 6 is defined without loft in any region, and this design degrades to the original one II-2. So, it is a part of the design but also provides a reference to others in type II. Under the expansion, the seventh datum shows a negative pressure value, which means there may be cavitation. This is because the fluid at a constant flow rate is fully developed before entering the bend, and it has to expand to fill the space caused by the geometry changes. Under this circumstance, the negative pressure region comes into existence.

Some useful information can be obtained in Table 6, but it cannot produce a recommendation. Therefore, the main effect plot, denoting how the feature affects the pressure drop under expansion and contraction condition, was carried out, and the main factor plot is presented in Figure 19. The column of the plot corresponds to regions $\mathrm{i}$, ii, and iii, and is accompanied by the row that is the mean value of pressure drop. The upward line means the factor contributes positively to the increase of the pressure drop. Generally speaking, the steeper the slope of the line, the greater the magnitude of the main effect. It is obvious that the loft feature in region $i$ and ii is beneficial to reduce pressure drop, and the loft applied in region $i$ has the most obvious effect on the pressure drop, while that loft in region iii has a negative influence. It is also important that whether in expansion or contraction, these three factors have the same effect. So it is clear that applying lofted geometry to region $i$ and ii can cause less pressure loss for both two circumstances and will not cause contradictory effects, and the eighth design was adopted as the optimal design finally.
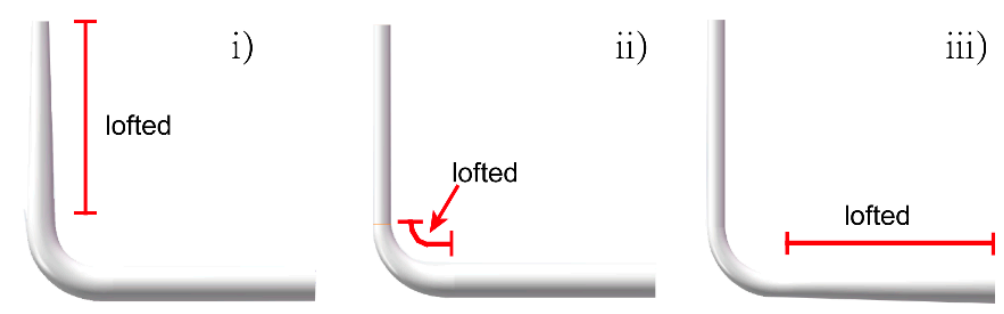

Figure 18. Lofted features applied in three different regions.

Table 6. Results of full factorial design for L-i, -ii, and -iii.

\begin{tabular}{cccccc}
\hline \multirow{2}{*}{ No. } & \multicolumn{3}{c}{ Coded Level } & \multicolumn{2}{c}{ Pressure Drop/Bar } \\
\cline { 2 - 6 } & i & ii & iii & Expansion & Contraction \\
\hline 1 & 0 & 0 & 1 & 1.103819 & 1.867616 \\
2 & 0 & 1 & 1 & 0.800478 & 1.524632 \\
3 & 1 & 1 & 1 & 0.244439 & 0.915409 \\
4 & 0 & 1 & 0 & 0.629615 & 1.352625 \\
5 & 1 & 0 & 1 & 0.19536 & 0.882602 \\
6 & 0 & 0 & 0 & 0.802054 & 1.28754 \\
7 & 1 & 0 & 0 & -0.01284 & 0.648752 \\
8 & 1 & 1 & 0 & 0.075031 & 0.739965 \\
\hline
\end{tabular}




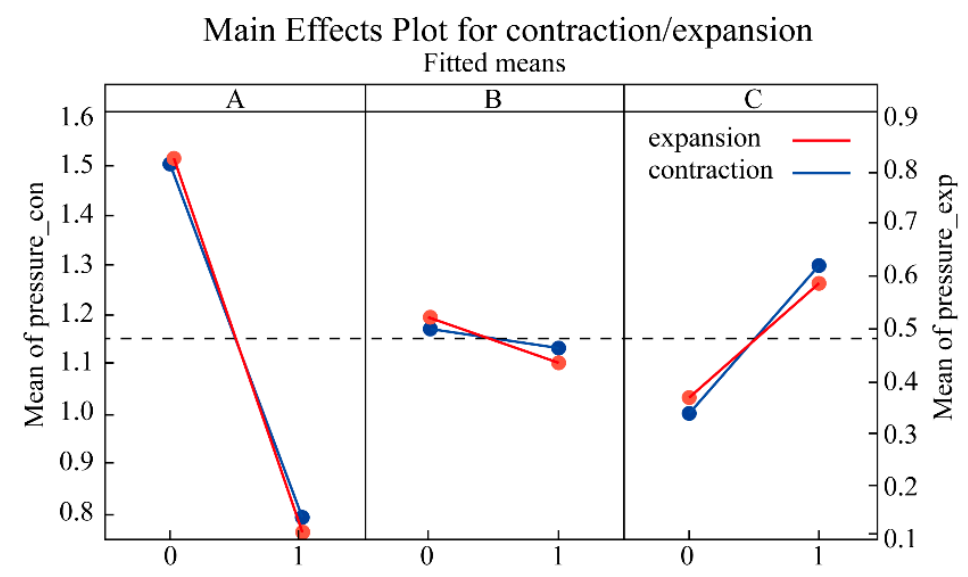

Figure 19. Main effect plot for expansion and contraction.

As for passage III, the idea is quite the same as that used for the passage I, but the difference is the $3 \mathrm{D}$ curve which is used as a mean of smooth transition.

Based on the analysis above, the performance for the three newly designed channel in the whole range of flow rate was compared and the result is given in Figure 20. Here, the nondimensioned pressure drop $\Delta \mathrm{P}_{\mathrm{n}}$ is obtained by dividing the pressure drop using $\varrho \mathrm{v}^{2} / 2$, in which $\varrho$ is the density of the fluid and $\mathrm{v}$ is the velocity at the maximum flow rate over reference flow area at the inlet. The flow rate is transferred to dimensionless one by $q / \mathrm{Q}$, where $\mathrm{Q}$ is the maximum flow rate of $25 \mathrm{~L} / \mathrm{min}$. The improvement is obvious, and the largest pressure reduction can reach up to more than $90 \%$ in Lo-110/E. It is followed by a smooth 3D curve with $40.8 \%$, and the next is Lo-110/C and C-1/1.5 with $38.7 \%$ and $23.1 \%$ respectively. Even Lo-110/E has the best improvement performance, we do not think the Lo-110/E can work in practice, this is because this may lead to cavitation and cause damage to other components. However, it cannot be denied that the novel design can reduce pressure drop effectively.

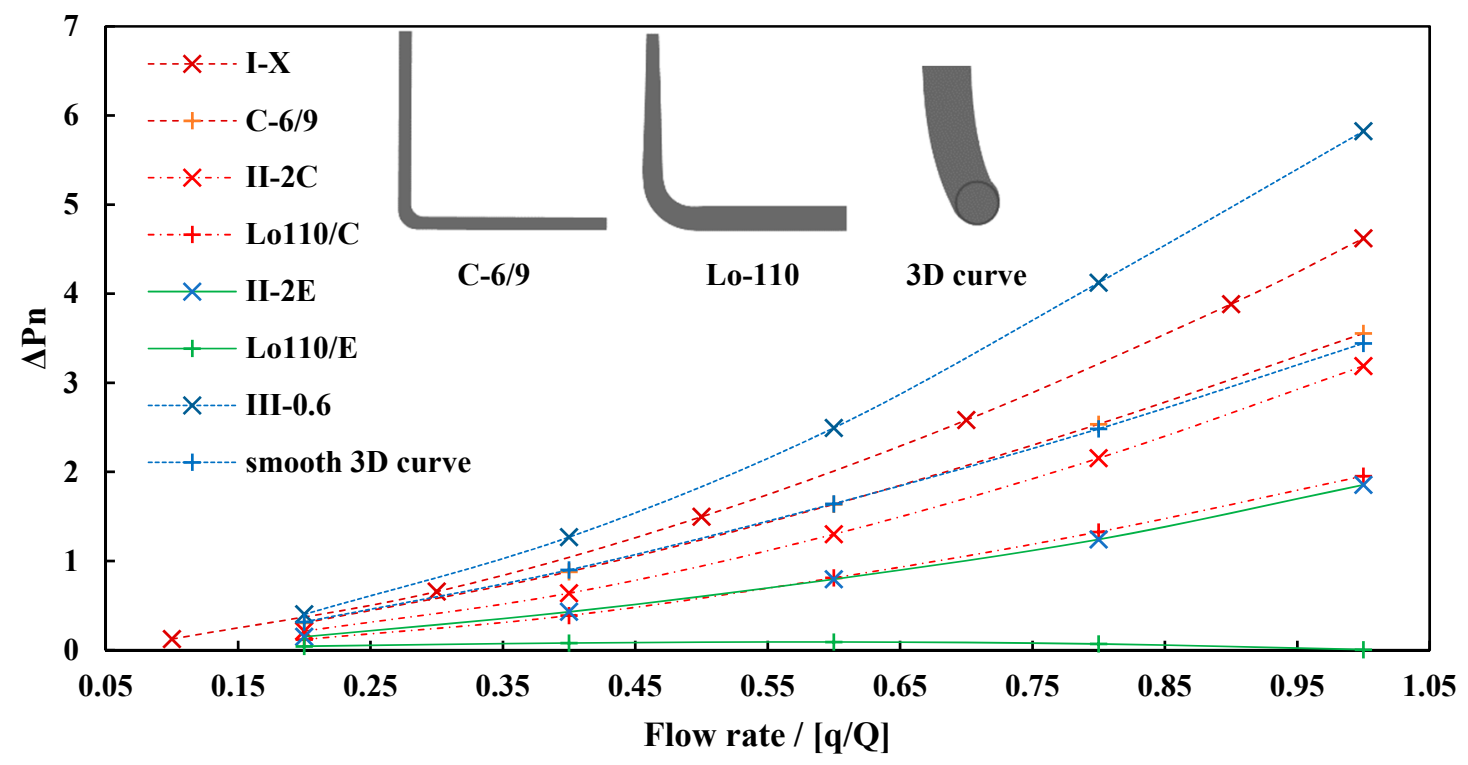

Figure 20. Comparison between traditional and optimized passages.

\subsection{Experimental Validation}

Based on the optimization, three optimal channels are adopted and a novel manifold was printed using AM technology. Shown in Figure 21, the lightweight design is applied and the channels are extracted as the pipe net. Compared to a bulk one, more than $70 \%$ reduction in the volume is achieved in the manifold. However, to build the tortuous channels using AM technology can be challenging 
because of the difficulties in postprocessing, especially for the manifolds in which feature size is larger than $10 \mathrm{~mm}$. Among various AM technologies, stereolithography (SLA) is the most suitable one to produce the design as it can print high-quality parts without the support structures, and was adopted in this article. However, this printed manifold is for testing only and cannot be directly used in industrial practice because of its inferior mechanical property, compared with conventional materials usually used like steel or aluminum.

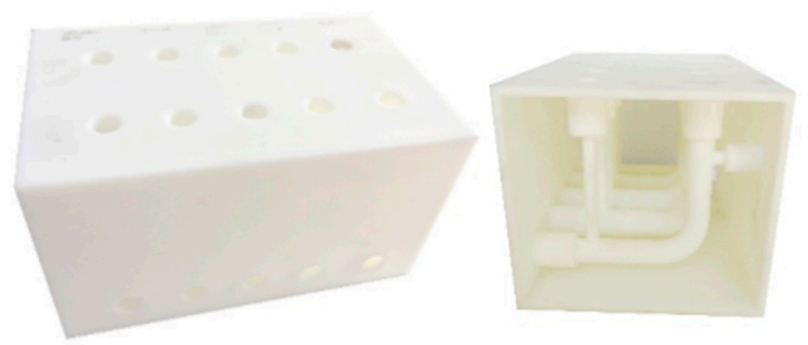

Figure 21. AMed manifold using stereolithography (SLA).

The novel manifold and the traditional one are tested on the experiment rig and the results are given in Figure 22. Out of our expectation, the maximum flow rate here is only $20 \mathrm{~L} / \mathrm{min}$ because of the mechanical property of the SLA manifold. However, the result shows the consistent trend with the simulation that passage II has a lower pressure drop compared to the passage I and III. Under the expansion condition, the pressure drop is quite stable and goes down slowly while the pressure drop rises as the flow rate increases under contraction condition. This corresponds well with the characteristic of the lofted channel. The other two novel channels also show distinct improvements when compared to the traditional ones. At a small flow rate, all channels show quite a small difference. This may be caused by the fact that the flow state is quite stable and the turbulence is not so acute at the low fluid velocity. As the flow rate increases, the turbulence gets tenser and the pressure drop increases. The figures tell that the large energy consuming and pressure loss are much alleviated using optimized channels, and the actual improvement can reach up to $55.2 \%$.

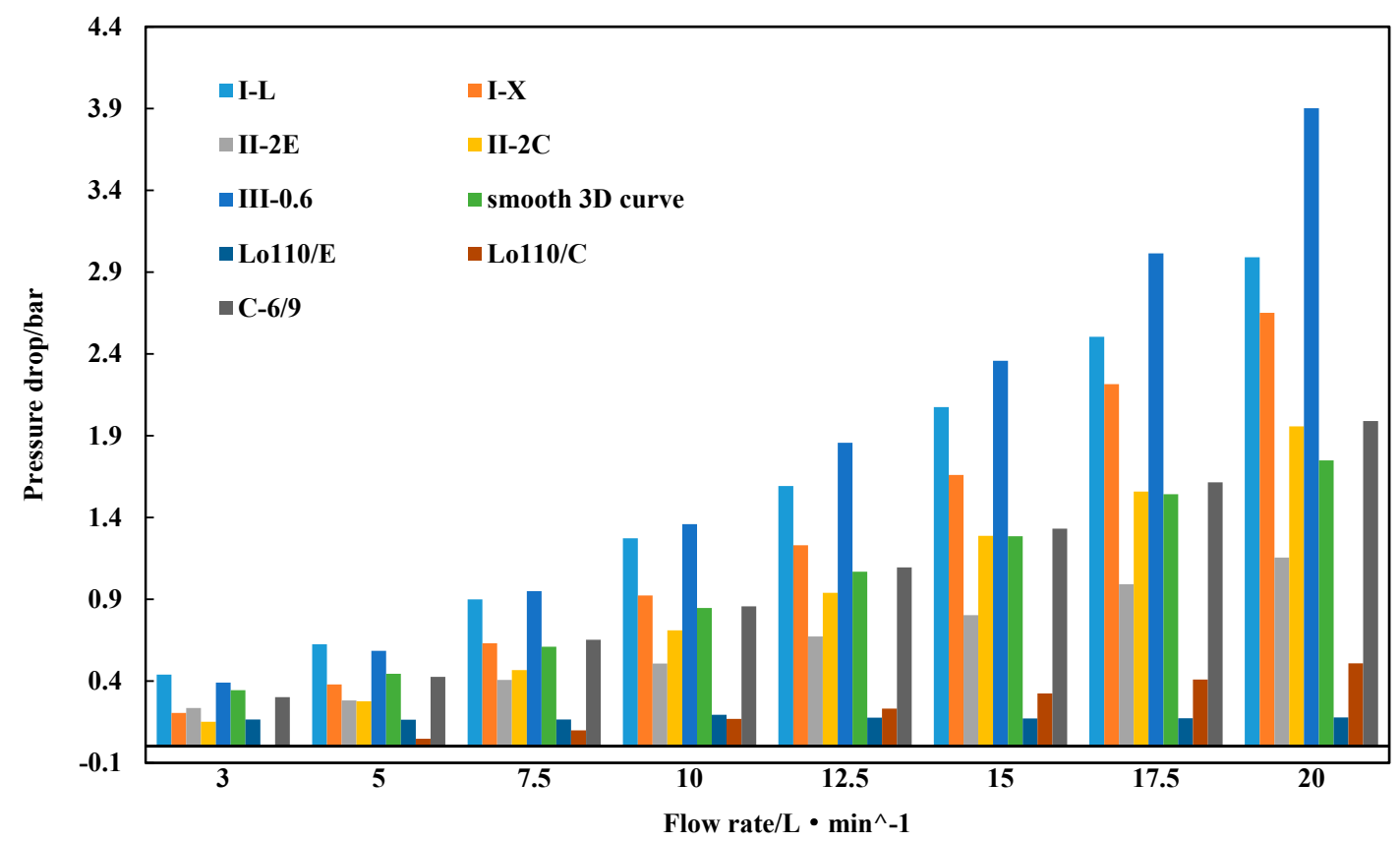

Figure 22. Comparison of pressure drop in traditional and novel channels. 


\section{Conclusions}

This work has mainly concentrated on the pressure loss in manifolds and proposed three novel passages with the aim of reducing the pressure loss which has not been explored in previous work. Combining experiments and simulations, the typical passages in traditional hydraulic manifolds are investigated. The abrupt changes in flow direction and effective flow area are the main reasons causing much pressure decline and energy dissipation. The intersection type mainly has effects on local pressure drop while the velocity changes account much for the friction loss. The intersection of two passages with different diameters has the least pressure loss but also has the risk of cavitation in expansion at a large flow rate, and some effective means are still needed.

To improve the energy efficiency of the manifold, the passages particularly applicable in AM technology are designed. The results have proved the potential of novel design to reduce pressure loss and energy consuming using AM technology. The pressure reduction can reach up to $55.2 \%$ in 3D smooth channels at the flow rate of $\sim 20 \mathrm{~L} / \mathrm{min}$. But the effect that surface roughness has on the pressure drop is inadequately discussed here because the limited manufacturing and postprocessing abilities make it hard to measure and control surface roughness of the inner surface. This problem should be discussed with further investigations. However, some basic rules may also be concluded from the research:

1. A smooth transition is encouraged to replace sharp $90^{\circ}$ bends, but the minimal curvature should be noticed to prevent the reduction of effective flow area.

2. Intersection out of a plane should be avoided. For offset intersection, the offset distance is supposed to be as small as possible, and the proper smooth passage is quite good to reduce pressure drop.

3. A moderate expansion in the channel is helpful to reduce the pressure loss but the ratio should be chosen carefully in avoid of cavitation.

4. 3D printing is a promising technology in hydraulic components manufacturing. By means of the $\mathrm{AM}$, the novel design that cannot be achieved by traditional manufacturing is realized and the improvement of energy saving performance can be guaranteed.

Though the optimization is based on a manifold, it may hopefully be useful and provide some basic understanding when designing internal channels using 3D printing in other fields.

Author Contributions: G.L. and J.-h.Z. conceived, designed, and realized the experiments and numerical analysis; K.Z. helped in the experiments; G.L. and K.Z. analyzed the data; G.L. wrote the paper; J.-h.Z., M.P., S.L. and R.D. revised it.

Funding: The work was funded by the National Natural Science Foundation of China (under Grant No. 51605425) and the NSFC-Zhejiang Joint Fund for the Integration of Industrialization and Informatization (under Grant No. U1509204).

Conflicts of Interest: The authors declare no conflicts of interest.

\section{Appendix A}

This part gives the information about the nodes in the different channel when the mesh ratio is 0.07 .

Table A1. Node information for the nine single channels.

\begin{tabular}{ccccccccc}
\hline Numbers & $\mathbf{1}$ & $\mathbf{2}$ & $\mathbf{3}$ & $\mathbf{4}$ & $\mathbf{5}$ & $\mathbf{6}$ & $\mathbf{7}$ & $\mathbf{8}$ \\
\hline Nodes & 210331 & 213916 & 219174 & 246866 & 304187 & 438177 & 215075 & 215321 \\
\hline Numbers & $\mathbf{9}$ & & & & & & & \\
\hline Nodes & 214987 & & & & & & \\
\hline
\end{tabular}


Table A2. Node information corresponding to Table 5 and Figures 14 and 15.

\begin{tabular}{ccccccccc}
\hline Numbers & $\mathbf{1}$ & $\mathbf{2}$ & $\mathbf{3}$ & $\mathbf{4}$ & $\mathbf{5}$ & $\mathbf{6}$ & $\mathbf{7}$ & $\mathbf{8}$ \\
\hline Nodes & 192516 & 192979 & 192548 & 189550 & 189977 & 192520 & 192384 & 192014 \\
\hline Numbers & $\mathbf{9}$ & $\mathbf{1 0}$ & $\mathbf{1 1}$ & $\mathbf{1 2}$ & $\mathbf{1 3}$ & $\mathbf{1 4}$ & $\mathbf{1 5}$ & $\mathbf{1 6}$ \\
\hline Nodes & 191971 & 191200 & 193635 & 191699 & 191537 & 192048 & 191204 & 193239 \\
\hline Numbers & $\mathbf{1 7}$ & $\mathbf{1 8}$ & $\mathbf{1 9}$ & $\mathbf{2 0}$ & $\mathbf{2 1}$ & $\mathbf{2 2}$ & $\mathbf{2 3}$ & $\mathbf{2 4}$ \\
\hline Nodes & 192144 & 192405 & 192321 & 191370 & 193871 & 192041 & 192509 & 192652 \\
\hline Numbers & $\mathbf{2 5}$ & & & & & & \\
\hline Nodes & 191097 & & & & & & &
\end{tabular}

Table A3. Node information corresponding to Figures 16 and 17.

\begin{tabular}{ccccccccc}
\hline Numbers & $\mathbf{1}$ & $\mathbf{2}$ & $\mathbf{3}$ & $\mathbf{4}$ & $\mathbf{5}$ & $\mathbf{6}$ & $\mathbf{7}$ & $\mathbf{8}$ \\
\hline Nodes & 283044 & 192493 & 191237 & 188705 & 187938 & 185632 & 181732 & 193804 \\
\hline Numbers & $\mathbf{9}$ & $\mathbf{1 0}$ & $\mathbf{1 1}$ & $\mathbf{1 2}$ & $\mathbf{1 3}$ & $\mathbf{1 4}$ & $\mathbf{1 5}$ & $\mathbf{1 6}$ \\
\hline Nodes & 193025 & 192485 & 190288 & 187235 & 187351 & 182645 & 194390 & 194210 \\
\hline Numbers & $\mathbf{1 7}$ & $\mathbf{1 8}$ & $\mathbf{1 9}$ & $\mathbf{2 0}$ & $\mathbf{2 1}$ & $\mathbf{2 2}$ & $\mathbf{2 3}$ & $\mathbf{2 4}$ \\
\hline Nodes & 192682 & 191669 & 188998 & 185664 & 185539 & 196059 & 195573 & 194876 \\
\hline Numbers & $\mathbf{2 5}$ & $\mathbf{2 6}$ & $\mathbf{2 7}$ & $\mathbf{2 8}$ & $\mathbf{2 9}$ & $\mathbf{3 0}$ & $\mathbf{3 1}$ & $\mathbf{3 2}$ \\
\hline Nodes & 192628 & 189964 & 187679 & 183744 & 196615 & 196821 & 196203 & 194795 \\
\hline Numbers & $\mathbf{3 3}$ & $\mathbf{3 4}$ & $\mathbf{3 5}$ & & & & & \\
\hline Nodes & 192149 & 189505 & 185194 & & & & & \\
\hline
\end{tabular}

\section{References}

1. Huber, J.E.; Fleck, N.A.; Ashby, M.F. The selection of mechanical actuators based on performance indices. Proc. R. Soc. Lond. Series A Math. Phys. Eng. Sci. 1997, 453, 2185-2205. [CrossRef]

2. Çelikkanat, H.; Şahin, E.; Kalkan, S. Integrating spatial concepts into a probabilistic concept web. In Proceedings of the International Conference on Advanced Robotics (ICAR), Istanbul, Turkey, 27-31 July 2015. [CrossRef]

3. Raibert, M.; Blankespoor, K.; Nelson, G.; Playter, R. BigDog, the rough-terrain quadruped robot. IFAC Proc. 2008, 41, 10822-10825. [CrossRef]

4. Semini, C.; Tsagarakis, N.G.; Guglielmino, E.; Focchi, M.; Cannella, F.; Caldwell, D.G. Design of HyQA hydraulically and electrically actuated quadruped robot. Proc. Inst. Mech. Eng. Part. I J. Syst. Control Eng. 2011, 225, 831-849. [CrossRef]

5. Quan, Z.; Quan, L.; Zhang, J. Review of energy efficient direct pump controlled cylinder electro-hydraulic technology. Renew. Sustain. Energy Rev. 2014, 35, 336-346. [CrossRef]

6. Zimmerman, J.D.; Pelosi, M.; Williamson, C.A.; Ivantysynova, M. Energy consumption of an LS excavator hydraulic system. In ASME 2007 International Mechanical Engineering Congress and Exposition; American Society of Mechanical Engineers: New York, NY, USA, 2007; pp. 117-126. [CrossRef]

7. Love, L.J. Estimating the Impact (Energy, Emissions and Economics) of the US Fluid Power Industry; University of North Texas Libraries, Digital Library: Denton, TX, USA, 2012.

8. Marani, P.; Martelli, M. Energy and control characteristics of a novel meter out hydraulic system for mobile applications. In ASME/BATH 2017 Symposium on Fluid Power and Motion Control; American Society of Mechanical Engineers: New York, NY, USA, 2017; ISBN 978-0-7918-5833-2.

9. Du, C.; Plummer, A.R.; Johnston, D.N. RETRACTED: Performance analysis of an energy-efficient variable supply pressure electro-hydraulic motion control system. Control Eng. Pract. 2016, 48, 10-21. [CrossRef]

10. Vukovic, M.; Sgro, S.; Murrenhoff, H. STEAM: A mobile hydraulic system with engine integration. In ASME/BATH 2013 Symposium on Fluid Power and Motion Control ASME; American Society of Mechanical Engineers: New York, NY, USA, 2013. 
11. Yuan, H.; Shang, Y.; Vukovic, M.; Wu, S.; Murrenhoff, H.; Jiao, Z. Characteristics of energy efficient switched hydraulic systems. JFPS Int. J. Fluid Power Syst. 2014, 8, 90-98. [CrossRef]

12. Murrenhoff, H.; Sgro, S.; Vukovic, M. An overview of energy saving architectures for mobile applications. In Proceedings of the 9th International Fluid Power Conference, Aachen, Germany, 26 March 2014.

13. Zhang, Y.; Chen, H.; Guo, K.; Zhang, X.; Eben Li, S. Electro-hydraulic damper for energy harvesting suspension: Modeling, prototyping and experimental validation. Appl. Energy 2017, 199, 1-12. [CrossRef]

14. Gong, J.; Zhang, D.; Guo, Y.; Liu, C.; Zhao, Y.; Hu, P.; Quan, W. Power control strategy and performance evaluation of a novel electro-hydraulic energy-saving system. Appl. Energy 2019, 233, 724-734. [CrossRef]

15. Zardin, B.; Cillo, G.; Rinaldini, C.; Mattarelli, E.; Borghi, M. Pressure losses in hydraulic manifolds. Energies 2017, 10, 310. [CrossRef]

16. Idelchik, I.E. Handbook of Hydraulic Resistance, 1st ed.; Hemisphere Publishing Corp.: Washington, DC, USA, 1960.

17. Murakami, M.; Shimizu, Y. Studies on fluid flow in three-dimensional bend conduits. JSME 1969, 12, 1369-1379. [CrossRef]

18. Rennels, D.C.; Hudson, H.M. Pipe Flow, A Practical and Comprehensive Guide; John Wiley \& Sons, Inc.: Hoboken, NJ, USA, 2012.

19. Oberlack, M. Similarity in non-rotating and rotating turbulent pipe flows. J. Fluid Mech. 1999, 379, 1-22. [CrossRef]

20. Wang, Z.; Örlü, R.; Schlatter, P.; Chung, Y.M. Direct numerical simulation of a turbulent $90^{\circ}$ bend pipe flow. Int. J. Heat Fluid Flow 2018, 73, 199-208. [CrossRef]

21. Zhang, H.; Tian, S.J.; Gao, Y.M.; Jia, C.Q. CFD-based optimal design of duct nets in hydraulic manifold block. J. Wuhan Univ. Technol. 2006, 28, 621-625.

22. Song, Z.A.; Yong'An, W. Research and analysis of the resistance characteristic of combined flow channel. In Proceedings of the 2009 International Workshop on Information Security and Application (IWISA 2009), Qingdao, China, 21-22 November 2009.

23. Abe, O.; Tsukiji, T.; Hara, T.; Yasunaga, K. Pressure Drop of Pipe Flow in Manifold Block. In 8th JFPS International Symposium on Fluid Power, Okinawa, Japan, 25-28 October, 2011; The Japan Fluid Power System Society: Okinawa, Japan, 2011; pp. 204-210.

24. Zardin, B.; Cillo, G.; Borghi, M.; D'Adamo, A.; Fontanesi, S. Pressure losses in multiple-elbow paths and in v-bends of hydraulic manifolds. Energies 2017, 10, 788. [CrossRef]

25. Kruth, J.-P.; Leu, M.; Nakagawa, T. Progress in additive manufacturing and rapid prototyping. CIRP Ann. Manuf. Technol. 1998, 47, 525-540. [CrossRef]

26. Gibson, I.; Rosen, D.W.; Stucker, B. Additive Manufacturing Technologies—Rapid Prototyping to Direct Digital Manufacturing; Springer: Berlin, Germany, 2014; ISBN 978-1-4419-1119-3.

27. Frazier, W.E. Metal additive manufacturing: A review. J. Mater. Eng. Perform. 2014, 23, 1917-1928. [CrossRef]

28. Tofail, S.A.M.; Koumoulos, E.P.; Bandyopadhyay, A.; Bose, S.; O'Donoghue, L.; Charitidis, C. Additive manufacturing: Scientific and technological challenges, market uptake and opportunities. Mater. Today 2018, 21, 22-37. [CrossRef]

29. Chhabra, M.; Singh, R. Rapid casting solutions: A review. Rapid Prototyp. J. 2011, 17, 328-350. [CrossRef]

30. Sachs, E.; Cima, M.; Cornie, J. Three-dimensional printing: Rapid tooling and prototypes directly from a CAD model. CIRP Ann. Manuf. Technol. 1990, 39, 201-204. [CrossRef]

31. Saunders, M. How AM Could Disrupt Your Market. Available online: https://www.renishaw.com/en/ additive-impact-part-2-how-am-could-disrupt-your-market--37551 (accessed on 27 March 2019).

32. Cooper, D.E.; Stanford, M.; Kibble, K.A.; Gibbons, G.J. Additive manufacturing for product improvement at Red Bull technology. Mater. Des. 2012, 41, 226-230. [CrossRef]

33. Schmelzle, J.; Kline, E.V.; Dickman, C.J.; Reutzel, E.W.; Jones, G.; Simpson, T.W. (Re)designing for part consolidation: Understanding the challenges of metal additive manufacturing. J. Mech. Des. 2015, 137. [CrossRef]

34. Qian, J.-Y.; Chen, M.-R.; Liu, X.-L.; Jin, Z.-J. A numerical investigation of the flow of nanofluids through a micro Tesla valve. J. Zhejiang Univ. Sci. A 2019, 20, 50-60. [CrossRef]

35. Rohsenow, W.M.; Hartnett, J.P.; Cho, Y.I. Handbook of Heat Transfer; McGraw-Hill: New York, NY, USA, 1998.

36. Gandhi, M.S.; Ganguli, A.A.; Joshi, J.B.; Vijayan, P.K. CFD simulation for steam distribution in header and tube assemblies. Chem. Eng. Res. Des. 2012, 90, 487-506. [CrossRef] 
37. Agrawal, Y.; Talbot, L.; Gong, K. Laser anemometer study of flow development in curved circular pipes. J. Fluid Mech. 1978, 85, 497-518. [CrossRef]

38. Sudo, K.; Sumida, M.; Hibara, H. Experimental investigation on turbulent flow in a circular-sectioned 90-degree bend. Exp. Fluids 1998, 25, 42-49. [CrossRef]

39. Kalpakli, A.; Örlü, R.; Alfredsson, P.H. Vortical patterns in turbulent flow downstream a $90^{\circ}$ curved pipe at high Womersley numbers. Int. J. Heat Fluid Flow 2013, 44, 692-699. [CrossRef]

(C) 2019 by the authors. Licensee MDPI, Basel, Switzerland. This article is an open access article distributed under the terms and conditions of the Creative Commons Attribution (CC BY) license (http://creativecommons.org/licenses/by/4.0/). 